\title{
Evaluation of pediatric patients with an oncologic emergency: Single center experience
}

\author{
Ahmet Çağrı Çiftçi Serhan Küpeli, Gülay Sezgin, İbrahim Bayram \\ Department of Pediatric Oncolog/Pediatric Bone Marrow Transplantation, Çukurova University, Faculty of Medicine, \\ Adana, Turkey.E-mail: serhankupeli@cu.edu.tr \\ Received: 10th August 2017, Revised: 3rd April 2018, Accepted: 8th April 2018
}

SUMMARY: Çiftçi AÇ, Küpeli S, Sezgin G, Bayram İ. Evaluation of pediatric patients with an oncologic emergency: Single center experience. Turk J Pediatr 2018; 60: 660-668.

In this study, we aimed to prospectively analyze the emergency diagnosis and clinical characteristics of the oncologic emergency patients admitted, as well as to evaluate the approach considerations to the cases, their treatment, patient outcomes and the prognostic factors.

A total of 88 oncologic emergency patients (90 oncologic emergency hospitalizations), except for febrile neutropenia, were admitted to the pediatric oncology department between July 2014 and December 2015.

A total of 30 cases of tumor lysis syndrome, 19 hyperleukocytosis, 11 convulsion, 7 spinal cord compression, 7 hemorrhagic cystitis, 9 pleural effusion, 6 vena cava superior syndrome, 4 typhlitis, 3 anuria, 4 gastrointestinal bleeding, 3 hypercalcemia, 4 acute respiratory distress syndrome, 2 acute massive hepatomegaly and 1 increased intracranial pressure were identified. Sixty-five $(72.2 \%)$ of the study patients had a primary oncologic disease, whereas 25 (27.8\%) had a recurring condition. The most common presenting complaint was fever (23.3\%) followed by breathing difficulties (22.2\%). Fifty-nine patients $(65.6 \%)$ recovered and were discharged home, while 31 cases $(34.4 \%)$ died.

Tumor lysis syndrome was the most frequently encountered emergency condition in the pediatric oncology patient group. Oncologic emergencies need to be approached quickly and systematically in the light of current scientific knowledge.

Key words: pediatric oncologic emergencies, childhood, tumor lysis syndrome.

Pediatric oncologic emergencies comprise the cases of acute and potentially life-threatening complications that directly or indirectly emerge due to the primary disease itself or its treatment. Pediatric oncologic emergencies can be further classified into subgroups such as metabolic emergencies, neurological emergencies, thoracic emergencies, gastrointestinal emergencies, urogenital emergencies, acute respiratory distress syndrome (ARDS) and febrile neutropenia. ${ }^{1}$

Frequently encountered metabolic emergencies include tumor lysis syndrome (TLS), hyperleukocytosis, hypercalcemia and hyponatremia, TLS being the most common. TLS is commonly seen in patients with acute leukemia or Burkitt-type non-Hodgkin lymphomas (NHL), in whom tumor burden is high, cellular turnover is rapid, chemotherapeutic susceptibility is high and leukocyte counts are elevated. ${ }^{2}$ Between $20-50 \%$ of these cases eventually die unless TLS is appropriately diagnosed and treated. ${ }^{3}$ The most commonly encountered neurological emergencies include spinal cord compression, convulsions, cerebrovascular accidents, increased intracranial pressure and improper intrathecal drug administrations. ${ }^{4}$ Vena cava superior syndrome (VCSS), pleural effusion, pericardial effusion, cardiac tamponade, massive hemoptysis, pneumothorax and pneumomediastinum are included in thoracic emergencies. Among the gastrointestinal emergencies are typhlitis (neutropenic enterocolitis, necrotizing 
enterocolitis, ileocecal syndrome), acute massive hepatomegaly, pancreatitis and veno-occlusive disease. ${ }^{5}$ Hemorrhagic cystitis, oliguria/ anuria and hypertension are considered as urogenital emergencies. ${ }^{6,7}$ The leading cause of mortality and morbidity among children under chemotherapy is infections. The patients get infected most commonly during the neutropenic intervals. ${ }^{8}$

Although the basic principles of the approach to and treatment of such cases are similar among different institutions, clinical management practices may vary greatly. In this study, we aimed to prospectively evaluate the emergency diagnosis, clinical characteristics, treatment, outcome and prognostic factors of the oncologic emergency patients admitted to the Pediatric Oncology Department of Çukurova University Medical School between 2014 and 2015.

\section{Material and Methods}

Patients admitted to the Pediatric Oncology Department of Çukurova University Medical School between June 2014 and December 2015, in whom at least one oncologic emergency condition (e.g., metabolic, neurological, thoracic, gastrointestinal or urogenital emergencies, or ARDS) was detected, were prospectively evaluated. Patients admitted for febrile neutropenia were excluded, in that, febrile neutropenia is a separate clinical entity and lies within the scope of another discipline. The study was approved by the Ethics Board for Medical Research (18 July 2014, approval number: 2014-7/33).

Upon gathering informed consent, the patients' age, gender, oncologic diagnoses, oncologic emergency diagnoses, admission dates to the emergency department, symptoms of presentation, clinical features, physical examination findings, laboratory results, imaging results, treatments and outcomes were recorded. The patients' medical records and oncologic follow-up charts were referred in order to obtain and record the detailed information regarding the following: histopathological diagnosis and stage of their primary disease, date of primary diagnosis, presenting symptoms at the time of primary diagnosis, chemotherapy protocols administered up to date and date of recurrence, if any. The patients were evaluated on a daily basis during their hospital stay, and the data regarding the laboratory studies, imaging studies, recruited treatments and clinical responses were recorded.

TLS was classified as laboratory TLS or clinical TLS, according to the CarioBishop classification. ${ }^{9}$ The cases with at least two of the Cario-Bishop laboratory TLS criteria (hyperuricemia, hyperkalemia, hyperphosphatemia, hypocalcemia) were identified as laboratory TLS. The cases showing one or more of the following: acute kidney failure (AKF), cardiac arrhythmia/sudden cardiac death or convulsion in addition to the above-defined laboratory TLS findings were identified as clinical TLS. All patients with TLS were hydrated with potassium free I.V. $1 / 2$ saline solution at $3000 \mathrm{ml} / \mathrm{m}^{2}$. During the course of hydration, fluid input-output was closely monitored to maintain the urinary output and urine density at $3-5 \mathrm{ml} / \mathrm{kg} / \mathrm{h}$ and less than 1010 , respectively. Intravenous furosemide $(0.5-1 \mathrm{mg}$ / $\mathrm{kg} / \mathrm{dose}$ ) was given to patients with insufficient urinary outputs despite the hydration therapy. The hydration fluid was supplemented with bicarbonate (50-100 $\mathrm{mEq} / \mathrm{L}$ ) in order to establish urinary alkalinization and to increase uric acid solubility. Urinary $\mathrm{pH}$ was maintained at 7-7.5. Blood samples were taken and sent to the laboratory for $\mathrm{Na}, \mathrm{K}$, urea, uric acid, phosphorus and calcium measurements once daily, or more frequently, as required depending on the patients' initial leukocyte counts and tumor loads. All patients with TLS were given the xanthine oxidase inhibitor, allopurinol, orally at $10 \mathrm{mg} / \mathrm{kg} /$ day or $300 \mathrm{mg} / \mathrm{m}^{2} /$ day divided into 2 or 3 equal doses, so as to decrease uric acid production. Rasburicase, a recombinant urate oxidase enzyme, was administered in a selected group of patients at $0.2 \mathrm{mg} / \mathrm{kg} /$ day, once or twice daily. To prevent secondary hyperkalemia due to TLS, I.V. hydration was performed without potassium. The patients with severe hyperkalemia $(>6 \mathrm{mEq} / \mathrm{L})$ or demonstrating signs of hyperkalemia on the electrocardiogram (ECG) were managed with potassium-lowering interventions (Ca gluconate, $\mathrm{Na}$ bicarbonate, insulin). The patients were given low-phosphate diets and hydration to prevent hyperphosphatemia secondary to TLS. Symptomatic hypocalcemic patients secondary to TLS were given $0.5-1 \mathrm{ml} / \mathrm{kg} \mathrm{I.V}$. bolus Ca gluconate (given within 5-10 minutes) under close cardiac monitorization for bradycardia. 
The patients in whom electrolyte imbalance persisted, urinary output was inadequate or AKF occurred despite all given hydration therapies and symptomatic treatments, underwent hemodiafiltration (HDF).

The patients with peripheral leukocyte counts greater than $100,000 / \mathrm{mm}^{3}$ were included in the hyperleukocytosis group and received I.V. hydration therapy at $3,000 \mathrm{ml} / \mathrm{m}^{2}$, alkaline fluids and allopurinol and leukocyte apheresis if necessary. Patients with spinal cord compression were evaluated with MRI as soon as possible and a pre-diagnosis for the primary disease was established. For the symptomatic patients, 1-2 $\mathrm{mg} / \mathrm{kg}$ dexamethasone was commenced and maintained at $0.5 \mathrm{mg} / \mathrm{kg} /$ dose every 6 hours. For patients diagnosed with VCSS, the head side of the bed was elevated and oxygen was supplemented with reservoir masks. Fluid intake was restricted in order to prevent cardiac failure, and I.V furosemide $(0.5-1 \mathrm{mg} / \mathrm{kg})$ was given to the cases with fluid overload. The patients with a suspicion of typhlitis were evaluated using standing direct abdominal radiographs, abdominal USG and abdominal CT. Oliguria and anuria were defined as having a urinary output of $<1 \mathrm{ml} / \mathrm{kg} / \mathrm{h}$ and having no urinary output at all, respectively. Berlin definition for ARDS was used in our study. ${ }^{10}$

SPSS 16.0 software was used for statistical analyses. Descriptive statistical expressions (median, percentage, frequency) were used for the majority of the calculations. Student- $t$ and Mann-Whitney $U$ tests were recruited to evaluate the statistical significance of the differences between groups with numerical variables showing and not showing a normal distribution, respectively. Non-numerical variables were analyzed using the Chi-square test. Statistical significance was defined as having a p-value lower than 0.05 .

\section{Results}

This study included a total of 88 patients with an oncologic emergency condition diagnosed between June 2014 and December 2015, except for febrile neutropenia. A total of 90 hospitalizations occurred during the study period. Two cases developed two distinct emergency conditions each, at different times. For 17 patients, there were more than one concurrent oncologic emergency conditions.
A total of 110 emergency conditions were detected during the study period. Coexistence of hyperleukocytosis and TLS was detected in 6 patients; hyperleukocytosis, TLS and hypercalcemia in 2 patients; hyperleukocytosis and pleural effusion in 1 patient; convulsion and ARDS in 1 patient; spinal cord compression and convulsion in 2 patients; TLS and pleural effusion in 1 patient; TLS, massive hepatomegaly and ARDS in 1 patient; TLS and VCSS in 2 patients; VCSS and pleural effusion in 1 patient. Of the patients included in the study, 59 were male $(67 \%)$ and 29 were female $(33 \%)$, with a male to female ratio of 2 .

The number of the newly diagnosed oncology patients was 362 during the study period. An oncologic emergency condition was detected in $63(17.4 \%)$ of these patients, either at the time of diagnosis or during their follow-up. Among the presenting complaints of all oncologic patients, fever and breathing difficulties were the most commonly encountered (Fig. 1).

TLS was present at the time of diagnosis in 26 of the 362 newly diagnosed oncology patients $(7.2 \%)$. Of these 362 naïve patients, 42 were diagnosed with ALL (11.6\%) and 42 with NHL (11.6\%). TLS developed in $26.2 \%$ (11 patients) and $28.6 \%$ (12 patients) of these newly diagnosed ALL and NHL cases, respectively.

TLS was the most prevalent among all of the oncologic emergency conditions studied. A total of 30 TLS cases were identified during the study. Cases of hospitalization for TLS comprised $33.3 \%$ of the total oncologic

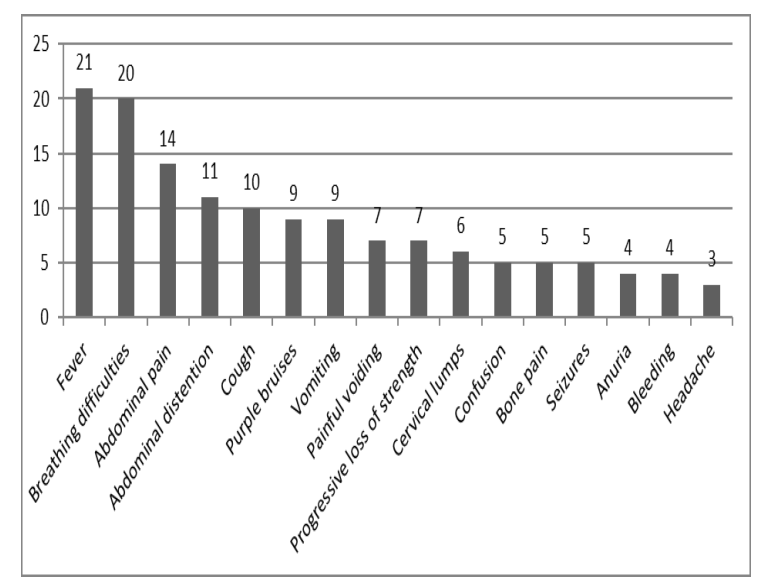

Fig. 1. Presenting Complaints. 
Table I. Characteristics of the Patients with Laboratory TLS.

\begin{tabular}{ccccccccccc}
\hline P. no & Diagnosis & Age & Gender & P/R & U.A. & K & P & Ca & Treatment & Outcome \\
\hline 1 & NHL & 16 & M & P & 8.3 & 4 & 2.6 & 8 & H,Alk,Alp & Discharged \\
2 & NHL & 17 & M & P & 8.1 & 4.3 & 5.1 & 8.3 & H,Alk,Alp & Discharged \\
3 & ALL & 8 & M & P & 7.9 & 4.4 & 4.8 & 9.5 & H,Alk,Alp & Discharged \\
4 & ALL & 11 & F & R & 15.3 & 6.1 & 2.8 & 19.6 & H,Alk,Alp & Ex \\
5 & ALL & 1 & M & P & 8.1 & 3.2 & 1.7 & 8.2 & H,Alk,Alp,Leuk & Discharged \\
6 & ALL & 8 & M & P & 8.9 & 3.9 & 5.5 & 10.1 & H,Alk,Alp,Ras & Discharged \\
7 & ALL & 9 & M & P & 8.2 & 3.8 & 4.1 & 7.8 & H,Alk,Alp & Discharged \\
8 & ALL & 7 & F & R & 8.5 & 4.2 & 3.8 & 7.7 & H,Alk,Alp & Discharged \\
9 & ALL & 13 & F & P & 7.8 & 4 & 4.8 & 9.4 & H,Alk,Alp & Discharged \\
10 & NHL & 8 & M & P & 15.8 & 4.4 & 14 & 4.2 & H,Alk,Alp,Hdf & Discharged \\
11 & ALL & 12 & F & P & 9.2 & 3.7 & 6.2 & 6.6 & H,Alk,Alp & Discharged \\
12 & ALL & 10 & M & P & 9.4 & 3.2 & 2.5 & 7.1 & H,Alk,Alp,Leuk & Discharged \\
\hline
\end{tabular}

P.no: Patient no, NHL: Non-Hodgkin lymphoma, ALL: Acute lymphoblastic leukemia, P: Primary, R: Recurrent, Ex: Exitus, H: Hydration, Alk: Alkalization, Alp: Allopurinol, Ras: Rasburicase, Hdf: Hemodiafiltration, Leuk: Leukapheresis

emergency hospitalizations. Among the 30 TLS cases, 20 were male $(66.7 \%)$ and 10 were female $(33.3 \%)$ with a male to female ratio of 2 . Of these $30,26(86.7 \%)$ were newly diagnosed oncology patients, and there were 4 $(13.3 \%)$ TLS cases due to a recurrent disease. Considering these 4 recurrent patients with TLS separately; 2 were identified as laboratory TLS $(16.7 \%$ of the total of 12 laboratory TLS patients with either a recurrent or a primarily diagnosed disease) and 2 cases as clinical TLS $(11.1 \%$ of the total of 18 clinical TLS patients with either a recurrent or a primarily diagnosed disease). Having a recurring disease was not found to be of statistical significance in predicting the development of laboratory or clinical TLS $(\mathrm{p}=1.00)$.

Of the TLS patients, $12(40 \%)$ were identified as laboratory TLS and 18 (60\%) were identified as clinical TLS. All patients with TLS showed hyperuricemia. Besides, 19 patients $(63.3 \%)$ had hypocalcemia, 19

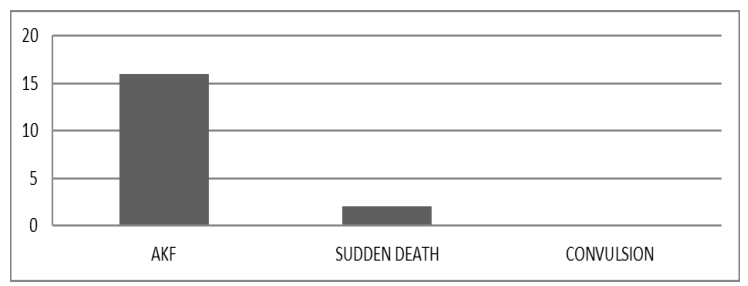

AKF: acute kidney failure

Fig. 2. Clinical manifestations in the patients with clinical TLS.
(63.3\%) had hyperphosphatemia and 4 $(13.3 \%)$ had hyperkalemia. Of the patients with laboratory TLS, 9 had ALL (75\%) and 3 had NHL (25\%) (Table I). All of these patients had hyperuricemia. Hypocalcemia, hyperphosphatemia and hyperpotassemia were detected in 8 (66.7\%), 6 (50\%) and $1(8.3 \%)$ of the laboratory TLS patients, respectively. One patient with laboratory TLS died (8.3\%) due to febrile neutropenic sepsis secondary to chemotherapy.

The primary diagnoses of the clinical TLS patients included NHL in 10 patients $(55.6 \%)$, ALL in $4(22.2 \%)$, neuroblastoma in $2(11.1 \%)$, Ewing sarcoma in $1(5.6 \%)$ and germ cell tumor in 1 patient $(5.6 \%)$ (Table II). An underlying hematological or lymphoid malignant condition was present in all of the laboratory TLS patients in our study, while, of the total of 18 patients with clinical TLS, 14 had (77.8\%) a hematological or lymphoid malignant disease. Regarding this, the two groups were statistically similar $(p=0.13)$. All clinical TLS patients had hyperuricemia. Other laboratory findings were hyperphosphatemia in 13 patients $(72.2 \%)$, hypocalcemia in 12 patients $(66.7 \%)$ and hyperpotassemia in 3 patients $(16.7 \%)$. The clinical components to establish the diagnosis of clinical TLS included AKF (present in 16 patients, $88.9 \%$ ) and sudden death (present in 2 patients, $11.1 \%$ ); nonetheless, none of our clinical TLS patients had convulsions (Fig. 2). Sudden death due to TLS occurred 
Table II. Characteristics of the Patients with Clinical TLS.

\begin{tabular}{|c|c|c|c|c|c|c|c|c|c|c|c|}
\hline P.no & Diagnosis & Age & Gender & $\mathrm{P} / \mathrm{R}$ & U.A & K & $\mathrm{P}$ & $\mathrm{Ca}$ & $\mathrm{CM}$ & Treatment & Outcome \\
\hline 1 & NHL & 4 & M & $\mathrm{P}$ & 14.3 & 5.2 & 25.3 & 4.3 & AKF & H,Alk,Alp,Hdf & Ex \\
\hline 2 & NHL & 1 & M & $\mathrm{P}$ & 11.4 & 9.2 & 4.4 & 7.4 & $\begin{array}{c}\text { Sudden } \\
\text { death }\end{array}$ & H,Alk,Alp & Ex \\
\hline 3 & NHL & 4 & M & $\mathrm{P}$ & 32 & 3.9 & 4.9 & 8.6 & $\mathrm{AKF}$ & $\begin{array}{l}\text { Alp, Hdf } \\
\text { H,Alk,Alp, }\end{array}$ & Discharged \\
\hline 4 & ALL & 16 & M & $\mathrm{P}$ & 11 & 6.1 & 3.7 & 11.3 & $\mathrm{AKF}$ & $\begin{array}{l}\text { Ras,Hdf, } \\
\text { Leuk }\end{array}$ & Ex \\
\hline 5 & ALL & 14 & M & $\mathrm{P}$ & 9.8 & 4.6 & 7.6 & 4.7 & $\mathrm{AKF}$ & $\begin{array}{l}\text { H,Alk,Alp, } \\
\text { Hdf,Leuk }\end{array}$ & Discharged \\
\hline 6 & NHL & 18 & M & $\mathrm{R}$ & 20.4 & 3.5 & 2.9 & 8.3 & AKF & H,Alk,Alp,Ras & Discharged \\
\hline 7 & NBL & 1 & $\mathrm{~F}$ & $\mathrm{P}$ & 7.9 & 3 & 5.5 & 5.6 & $\mathrm{AKF}$ & H,Alk,Alp & Ex \\
\hline 8 & ALL & 7 & $\mathrm{~F}$ & $\mathrm{P}$ & 6.4 & 4.6 & 12 & 6 & $\mathrm{AKF}$ & H,Alk,Alp,Hdf & Discharged \\
\hline 9 & NHL & 4 & M & $\mathrm{P}$ & 17.2 & 4.3 & 5 & 9 & $\mathrm{AKF}$ & H,Alk,Alp,Hdf & Ex \\
\hline 10 & NHL & 10 & $\mathrm{~F}$ & $\mathrm{P}$ & 5.6 & 4 & 9.8 & 5.8 & $\mathrm{AKF}$ & H,Alk,Alp,Hdf & Ex \\
\hline 11 & NHL & 3 & M & $\mathrm{P}$ & 8.9 & 5 & 16.9 & 5.1 & $\mathrm{AKF}$ & H,Alk,Alp,Hdf & Discharged \\
\hline 12 & Ewing & 1 & $\mathrm{~F}$ & $\mathrm{P}$ & 10.4 & 6.1 & 2.1 & 10.5 & $\mathrm{AKF}$ & H,Alk,Alp & Ex \\
\hline 13 & NHL & 8 & M & $\mathrm{P}$ & 12.3 & 4.5 & 12.5 & 7.6 & AKF & H,Alk,Alp,Hdf & Discharged \\
\hline 14 & NHL & 10 & M & $\mathrm{P}$ & 19.6 & 3.8 & 4.8 & 13.2 & AKF & H,Alk,Alp,Hdf & Ex \\
\hline 15 & ALL & 8 & M & $\mathrm{P}$ & 31.4 & 4.3 & 5.2 & 8.4 & $\mathrm{AKF}$ & H,Alk,Alp,Hdf & Discharged \\
\hline 16 & NHL & 2 & $\mathrm{~F}$ & $\mathrm{P}$ & 8.7 & 4.3 & 5.2 & 7.9 & $\begin{array}{c}\text { Sudden } \\
\text { death }\end{array}$ & H,Alk,Alp & Ex \\
\hline 17 & GCT & 12 & $\mathrm{~F}$ & $\mathrm{P}$ & 8.3 & 2.9 & 4.2 & 7.8 & $\mathrm{AKF}$ & H,Alk,Alp & Discharged \\
\hline 18 & NBL & 4 & M & $\mathrm{R}$ & 11.2 & 5.9 & 7.5 & 7 & $\mathrm{AKF}$ & H,Alk,Alp & Ex \\
\hline
\end{tabular}

P.no: Patient no, NHL: Non-Hodgkin lymphoma, ALL: Acute lymphoblastic leukemia, P: Primary, R: Recurrent, Ex: Exitus, H: Hydration, Alk: Alkalization, Alp: Allopurinol, Ras: Rasburicase, Hdf: Hemodiafiltration, Leuk: Leukapheresis.

in 2 cases $(11.1 \%)$; whereas, 8 cases $(44.4 \%)$ died because of other primary-disease-related problems. Utilization of HDF in the cases of clinical TLS seemed to have beneficial effects; however, the mortality rates of the patients that did and did not receive HDF treatment $(45.4 \%$ and $71.4 \%)$ were not statistically different $(p=0.36)$. One patient in laboratory TLS group and two patients in clinical TLS group received rasburicase.

Ten patients with clinical TLS $(55.6 \%)$ and 1 patient with laboratory TLS $(8.3 \%)$ died, despite every effort and intervention. The mortality rates between the two groups were significantly different $(p=0.009)$.

Oncologic emergencies other than TLS are shown in Table III. When all patients with an oncologic emergency condition were considered, $44(67.7 \%)$ of the 65 oncologic emergency cases, whose emergency conditions were present during the primary disease course recovered and were discharged; whereas for the emergency cases with a recurring disease, the rates were $15(60 \%)$ out of 25 . Mortality and discharge rates were found to be statistically similar between these two patient populations $(\mathrm{p}=0.49)$.

Thirteen (43.4\%) of the 30 oncologic emergency patients with solid tumors, and $18(30 \%)$ of the 60 emergency patients with hematologic or lymphoid malignant conditions died. The difference between the mortality rates of the patients with solid tumors and those with hematologic/lymphoid malignancies was not statistically significant $(p=0.21)$.

\section{Discussion}

Among pediatric oncology patients, oncologic emergencies are commonly encountered and usually require hospitalization; nevertheless, there are a limited number of studies on this patient group. Although there are numerous studies in the literature regarding febrile neutropenia, data on the other oncologic emergencies are scarce. Here in this study, we aim to raise awareness on these conditions.

According to the 2002 data, of all age group 
Table III. Patients with Oncologic Emergencies Other than TLS.

\begin{tabular}{lcc}
\hline Oncologic emergency & Number & Outcome \\
\hline Hyperleukocytosis & 19 & 4 exitus, 15 discharge \\
Convulsion & 11 & 5 exitus, 6 discharge \\
Pleural effusion & 9 & 2 exitus, 7 discharge \\
Spinal cord compression & 7 & 1 exitus, 6 discharge \\
Hemorrhagic cystitis & 7 & 1 exitus, 6 discharge \\
VCSS & 6 & 3 exitus, 3 discharge \\
Typhlitis & 4 & 2 exitus, 2 discharge \\
GI bleeding & 4 & 3 exitus, 1 discharge \\
ARDS & 4 & 2 exitus, 2 discharge \\
Anuria & 3 & 1 exitus, 2 discharge \\
Hypercalcemia & 3 & 2 exitus, 1 discharge \\
Massive hepatomegaly & 2 & 2 exitus \\
Increased intracranial pressure & 1 & Exitus \\
\hline
\end{tabular}

VCSS: Vena cava superior syndrome, GI bleeding: Gastrointestinal bleeding, ARDS: Acute respiratory distress syndrome.

cancer patients throughout the world, $53.4 \%$ are male and $46.6 \%$ are female. ${ }^{11}$ In a study by Kerrouault et al., it was reported that $65 \%$ of cancer patients that were admitted to the emergency department were men and $35 \%$ were women. ${ }^{12}$ In another retrospective emergency department study by Yaylaci et al., it was found that $55.7 \%$ of oncology cases were male and $44.3 \%$ were female. ${ }^{13}$ The distribution reported by Swenson et al., on the other hand, was as $41 \%$ male and $59 \%$ female (14). Our study revealed that, of the oncologic emergency patients, 59 were male $(67.1 \%)$ and 29 were female $(32.9 \%)$, with a male to female ratio of 2 . In accordance with the majority of the previous literature, there were more male patients than females admitting to the oncologic emergency department. Our results demonstrate that there is a male predominance in oncologic emergency admissions, which is in parallel with the case for the adult oncologic patient population. This male predominance among the pediatric oncology cases may be partly attributable to the similar epidemiological situation regarding the adult patients, and also it may be a consequence of a possible positive discrimination favoring males, which is phenomenal in our country's socio-cultural atmosphere. The gender distribution of all the 362 newly diagnosed oncology patients in our department during our study period, which was between June 2014 and December 2015, was as 219 males (60.5\%) and 143 females (39.5\%).

As for the presenting complaints of the oncologic emergency patients in our study, fever $(n=21 ; 23.3 \%)$ and breathing difficulties $(n=20 ; 22.2 \%)$ were the most commonly encountered ones. Yaylac1 et al., in their emergency department study, reported that the most common presenting symptom was breathing difficulty (22\%). In their work, fever was considered as a constitutional symptom along with weight loss, fatigue, lack of appetite, poor general status and edema. In that study, the prevalence of the constitutional symptoms was reported to be $20 \%$ (13). In another emergency department study by Bozdemir et al., dyspnea was reported to be the second (17\%) and fever to be the fourth (13\%) most common presenting symptom among oncologic patients. ${ }^{14}$ Dyspnea was the fourth most frequent complaint reported in the Swenson et al. study. ${ }^{15}$ In accordance with the majority of the previous adult studies, fever was the most commonly encountered presenting symptom in our work. Although febrile neutropenia was not included in our study as an oncologic emergency, fever was the most frequent presenting complaint of the patients, which was suggestive of the possibility 
that the patients mainly might have had a primary fever due to the underlying oncologic condition. The patients with leukemia, in particular, had low absolute neutrophil counts with high blast cell numbers, which might have led to frequent infections. Similarly, fever was also present in the vast majority of the patients with typhlitis and ARDS.

TLS was the most commonly encountered oncologic emergency condition during our study $(n=30 ; 33.3 \%)$. Considering only the newly diagnosed oncologic cases during the study period, TLS was present in 26 patients $(7.2 \%)$ at the time of diagnosis. The majority of the cases with TLS in our study were the patients with a primary diagnosis of ALL or NHL. Our results reveal that TLS is an emergency condition that mainly occurs at the time of initial diagnosis, and is most prevalently seen in the patients with ALL and Burkitt-type lymphoma.

In a study by Wossman et al., the rate of TLS among the pediatric cases of Burkitt lymphoma and B-ALL group disease was been found to be $8.4 \% .{ }^{16}$ In our study, the prevalence of TLS at the time of initial diagnosis among the patients with NHL and ALL group disease $(n=84)$ was found to be $27.4 \%(n=23)$. The relatively higher TLS rates in our study, when compared with the previous literature, may in part be explained by the fact that our patients generally present late in the course or during the advanced clinical stage, for our hospital being a tertiary healthcare setting. Montesinos et al. ${ }^{17}$ treated a total of 72 AML patients with allopurinol and prophylactic IV hydration between 1980 and 2002. They reported that clinical and laboratory TLS had occurred in $5 \%$ and $12 \%$ of the patients, respectively, according to Cairo-Bishop Classification. ${ }^{17}$ Another trial studying high-risk NHL cases revealed laboratory and clinical TLS rates of $42 \%$ and $6 \%$, respectively. ${ }^{18}$ In our study, for the cases with TLS, clinical TLS prevailed within the NHL group; whereas, the majority of ALL patients developed laboratory TLS. In a study by Cairo et al. ${ }^{19} 218$ patients with a diagnosis of advanced Burkitt lymphoma/ leukemia underwent aggressive fluid therapy, urine alkalinization and allopurinol medication. The authors reported a TLS (laboratory and clinical TLS) rate of $16.1 \% .{ }^{19}$ Our results demonstrated that whether having a recurrent underlying disease or not was not a statistically significant predictor of laboratory or clinical TLS occurrence $(p=1.00)$. All patients with TLS in our study had hyperuricemia $(n=30$; $100 \%$ ), which is, according to our results, an indispensable laboratory finding in TLS, followed by hypocalcemia and hyperphosphatemia. Considering the laboratory manifestations of the patients with laboratory TLS and clinical TLS separately, hypocalcemia was the second most common finding among the cases with laboratory TLS ( $n=8$ out of $12 ; 66.7 \%$ ); whereas hyperphosphatemia was the second most common finding among the cases with clinical TLS ( $\mathrm{n}=13$ out of $18 ; 72.2 \%$ ), after hyperuricemia.

In our study, all 12 patients in the laboratory TLS group had either a hematologic or a lymphoid malignant disease $(100 \%)$. Of the 18 patients in the clinical TLS group, 14 (77.8\%) had a hematologic or a lymphoid malignant condition. There was no statistically significant difference between the two groups $(\mathrm{p}=0.13)$.

All patients with laboratory TLS were treated with hydration, alkalization and allopurinol, and TLS eventually resolved in all. One patient belonging to this group (8.3\%) died during the follow-up due to febrile neutropenia. The results of our study show that the patients with laboratory TLS completely recover with IV hydration, alkalization and allopurinol treatments. One patient with significant hyperphosphatemia underwent and benefitted from HDF.

Among the manifestations to establish the diagnosis of clinical TLS, AKF was present in 16 patients $(88.9 \%)$ and sudden death occurred in $2(11.1 \%)$; whereas convulsion did not occur in any patient. These results altogether indicate that AKF is the most frequent manifestation encountered in the clinical TLS patients. Ten patients with clinical TLS (55.6\%) and 1 patient with laboratory TLS (8.3\%) were lost despite all efforts and interventions. Clinical TLS was found to lead to significantly higher mortality rates than laboratory TLS $(p=0.009)$. The majority of the patients $(75 \%)$ who recovered from clinical TLS $(n=8)$ underwent $\operatorname{HDF}(n=6)$, which was suggestive of the fact that HDF might have a beneficial effect on the survival rates in this patient group. On the other hand, although HDF seemed to improve the course 
of clinical TLS, the difference between the mortality rates among the patients who did and did not undergo HDF was not statistically significant $(45.4 \%$ vs. $71.4 \% ; \mathrm{p}=0.36)$.

Continuous renal replacement therapy (CRRT) is an extracorporeal treatment method for the patients with renal dysfunction, in which fluid, electrolytes and small- and mid-sized solutes are continuously cleared out of the blood through a machine with a special pumping system. There are several types of CRRT according to the access sites (arteriovenous, venovenous), returning points of the replacement fluid (pre-dilution, post-dilution) and the principle mode of action (hemofiltration, hemodialysis, hemodiafiltration). Convection and diffusion mechanisms underlie the physicochemical basis for hemofiltration and hemodialysis, respectively; while for HDF, both mechanisms work. HDF is the most effective solute-clearing method among the others ${ }^{20}$, which makes it the method of choice in our hospital. The infrastructure and experienced staff needed for HDF are available in our institution.

In our study, $44(67.7 \%)$ of the 65 oncologic emergency cases, whose emergency conditions were present during the primary disease course recovered and were discharged; whereas as for the emergency cases with a recurring disease, the rates were $15(60 \%)$ out of 25 . Our study revealed that the mortality and discharge rates were found to be statistically similar between these two patient populations (i.e., patients with a primary condition and the patients with a recurring disease) $(p=0.49)$. Thirteen (43.4\%) of the 30 oncologic emergency patients with solid tumors and $18(30 \%)$ of the 60 emergency patients with hematologic or lymphoid malignant conditions died. Our study also showed that the difference between the mortality rates of the patients with solid tumors and those with hematologic/lymphoid malignancies was not statistically significant $(p=0.21)$. In our study, of the total 90 oncologic emergency admissions, 59 patients $(65.6 \%)$ were discharged and 31 patients (34.4\%) died. This high mortality rate suggests that the pediatric patients with an oncologic emergency diagnosis need to be approached quickly and systematically in the light of current scientific knowledge.

\section{REFERENCES}

1. Freedman JL, Rheingold SR, Fisher MJ. Oncologic emergencies. In: Pizzo AP, Poplack DG, (eds). Principles and Practice of Pediatric Oncology (7th ed). Philadelphia: Lippincott, Williams\&Wilkins, 2016: 967-991.

2. Li HC, Chung OK, Tam CJ, Chiu SY. Effective Prevention and Management of Tumor Lysis Syndrome in Children With Cancer: The Important Contributions of Pediatric Oncology Nurses. J Pediatr Oncol Nurs 2015; 32: 209-218.

3. Bose P, Qubaiah O. A review of tumor lysis syndrome with targeted therapies and the role of rasburicase. J Clin Pharm Ther 2011; 36: 299-326.

4. Sandberg DI, Bilsky MH, Kushner BH, et al. Treatment of spinal involvement in neuroblastoma patients. Pediatr Neurosurg 2003; 39: 291-298.

5. Davila ML. Neutropenic enterocolitis. Curr Opin Gastroenterol 2006; 22: 44-47.

6. Stillwell TJ, Benson RC Jr. Cyclophosphamide-induced hemorrhagic cystitis. A review of 100 patients. Cancer 1988; 61: 451-457.

7. West NJ. Prevention and treatment of hemorrhagic cystitis. Pharmacotherapy 1997; 17: 696-706.

8. Hughes WT, Armstrong D, Bodey GP, et al. 2002 guidelines for the use of antimicrobial agents in neutropenic patients with cancer. Clin Infect Dis 2002; 34: 730-751.

9. Cairo MS, Bishop M. Tumor lysis syndrome: New therapeutic strategies and classification. Br J Haematol 2004; 127: 3-11.

10. Ranieri VM, Rubenfeld GD, Thompson BT, et al ARDS Definition Task Force. Acute respiratory distress syndrome: The Berlin Definition. JAMA 2012; 307: 2526-2533.

11. İzmirli M, Altın S, Dernek BO, Ünsal M. SSK Okmeydanı Eğitim ve Araştırma Hastanesi Onkoloji Merkezi'nin 1999-2004 yılları kanser istatistikleri. Türk Onkoloji Dergisi 2007; 22: 172-182.

12. Kerrouault E, Denis N, Le Conte P, Dabouis G. Improving organization of care could reduce referrals of cancer patients to the emergency department. Prospective analysis of 123 patients. Presse Med 2007, 36(11 Pt 1): 1557-1562.

13. Yaylacı S, Topuzoğlu A, Karcığlu O. Acil servise başvuran kanser hastalarının klinik karakteristikleri ve bir yıllık sağkalımları. Uluslararası Hematoloji-Onkoloji Dergisi 2009; 19: 213-222.

14. Bozdemir N, Eray O, Eken C, Senol Y, Artac M, Samur M. Demographics, clinical presentations and outcomes of cancer patients admitted to the emergency department. Turk J Med Sci 2009; 39: 235-240.

15. Swenson KK, Rose MA, Ritz L, Murray CL, Adlis SA. Recognition and evaluation of oncology-related symptoms in the emergency department. Ann Emerg Med 1995; 26: 12-17. 
16. WössmanW, Schrappe M, Meyer U, Zimmermann $\mathrm{M}$, Reiter A. Incidence of tumor lysis syndrome in children with advanced stage Burkitt's lymphoma/ leukemia before and after introduction of profilactic use of urate oxidase. Ann Hematol 2003; 82: 160-165.

17. Montesinos P, Lorenzo I, Martin G, et al. Tumor lysis syndrome in patients with acute myeloid leukemia: Identification of risk factors and development of a predictive model. Haemotologica 2008; 93: 67-74.
18. Hande KR, Garrow GC. Acute tumor lysis syndrome in patients with highgrade non-Hodgkin's lymphoma. Am J Med 1993; 94: 133-139.

19. Cairo MS, Coiffier B, Reiter A, Younes A; TLS Expert Panel. Reccomendations for the evaluation of risk and profilaxis of tumour lysis syndrome (TLS) in adults and children with malignant diseases: An expert TLS panel consensus. Br J Haematol 2010; 149: 578-586.

20. Ronco C. Hemodiafiltration: Technical and clinical issues. Blood Purif 2015; 40(Suppl 1): 2-11. 